\title{
Extraordinary Optical Reflection from Annular Dielectric Nanorod Arrays
}

\author{
Suxia Xie*, Jiangiiamin Chen and Zhijian Li
}

School of Physics and Electronic Science, Hunan University of Science and Technology, Xiangtan 411201, P.R. China

\begin{abstract}
Responses of free standing subwavelength annular dielectric nanorod arrays are described. Extreme reflection peaks with high quality factor follow a multiple scattering approach, which can be explained by geometrical resonance. It is demonstrated that the value and the resonant frequency of the sharp geometric resonance modes can be tuned by geometric parameters such as the outer radius, inner radius of the annular nanorod array, and incidence angle sensitively, which can provide new regulating factors and guidance for the design of novel optical device using the dielectric material with a lower volume.
\end{abstract}

Keyword: Annular nanorod, Dielectric, Extraordinary Optical Reflection, Geometric resonance.

\section{INTRODUCTION}

Peirodic metallic subwavelength structures such as hole arrays in metal films [1,2], metallic nano particle array [3], metallic nanowire gratings [4], and metallic heterostructures [5-8] are investigated widely for the applications due to the strong interaction with light as a result of the plasmonic resonances. However, in nanometer scale, the metallic subwavelength structure is not the optimal selection due to intrinsic metal absorption.

A very sharp reflection peak with an even higher qualityfactor can be excited from a dielectric nanorod array, which is the geometric resonance [9-13]. It means that the dielectric nanorod array can reflect most light over the wavelength range, making this a promising material for even better applications in enhanced Raman scattering [14], fluorescence enhancement [15], nonlinear optics [16], and coherent light emission [17], multispectral imaging [18, 19], and optomechanics $[20,21]$.

In this paper, we propose an optical system of a single layer annular dielectric nanorod to analyze the geometric resonances. We clarify the dependence of the geometric resonance on the inner radius and the outer radius of the annular dielectric nanorods and the incidence angle, and explain it by the multiple scattering effects. The results furnish a new regulating method for the optical control and potential application for filters using a dielectric material.

\section{MATERIALS AND METHODS}

Finite difference time domain method (FDTD) is a numerical analysis technique used for modeling computational

*Address correspondence to this author at the School of Physics and Electronic Science, Hunan University of Science and Technology, Xiangtan 411201, P.R. China; Tel: +86 731 58291433; Fax: +86 731 58291641;

E-mail:_xiesuxia2013@163.com electrodynamics. The time dependent Maxwell's equations are discretized using central difference approximations to the space and time partial derivatives. When Maxwell's differential equations are examined, it can be seen that the change in the E-field in time (the time derivative) is dependent on the change in the $\mathrm{H}$-field across space (the curl). The analyzed structure is presented in Fig. (1). In our 2-dimension FDTD calculations [22], perfectly matched layer boundary conditions [23] are used at the top and bottom, and periodic boundary conditions are used on the left and right sides of the lattice due to the periodicity of the system. We simulate the structure with a computational window of $L_{x} \times L_{y}$ $=3 \mu \mathrm{m} \times 6 \mu \mathrm{m}$, where the structure in the $z$ direction is uniform and infinite. The structure is periodic and the periodicity is $p=3 \mu \mathrm{m}$. We send a Gaussian single pulse of light with a wide frequency profile and an incidence angle of $\theta$ illuminating the dielectric annular grating from the bottom of the lattice in the $x y$ cross section. Parameters of the annular nanorod are denoted as the outer radius $R_{I}$ and the inner radius $R_{2}$, as shown in Fig. (1). The relative dielectric constant of the dielectric annular nanorod is set $\varepsilon=3.9$ in the paper, and $\mathrm{SiN}_{\mathrm{x}}$ is a possible material for making annular nano-rods.

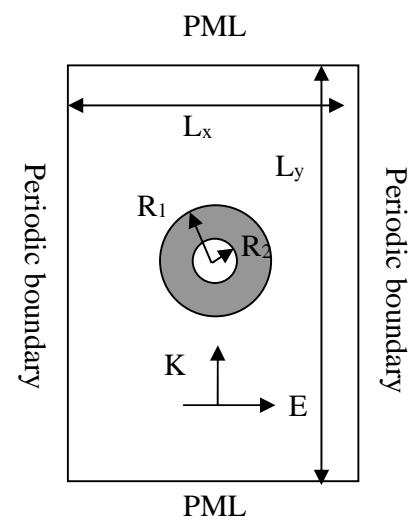

Fig. (1). A lattice of the free-standing dielectric annular nanorod grating illuminated from the bottom with an incidence angle $\theta$. 


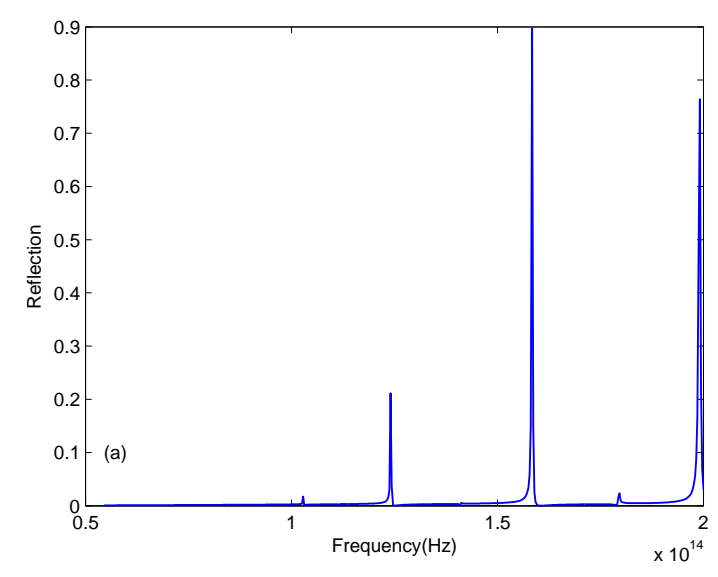

(a)

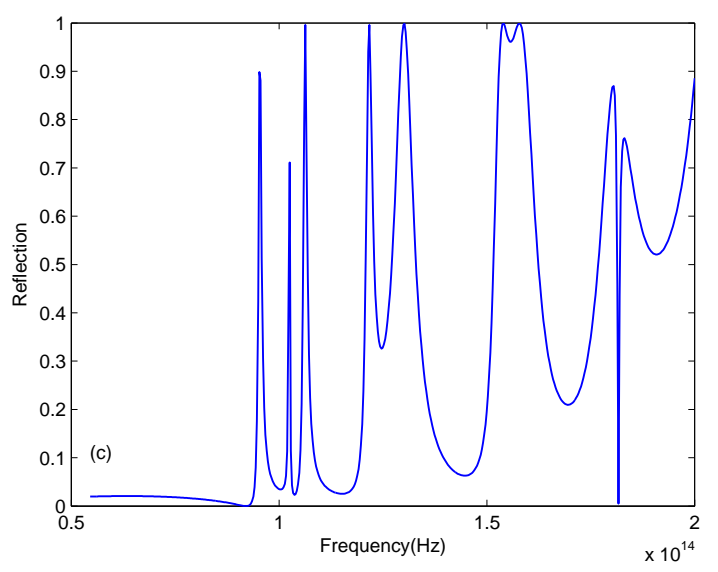

(c)

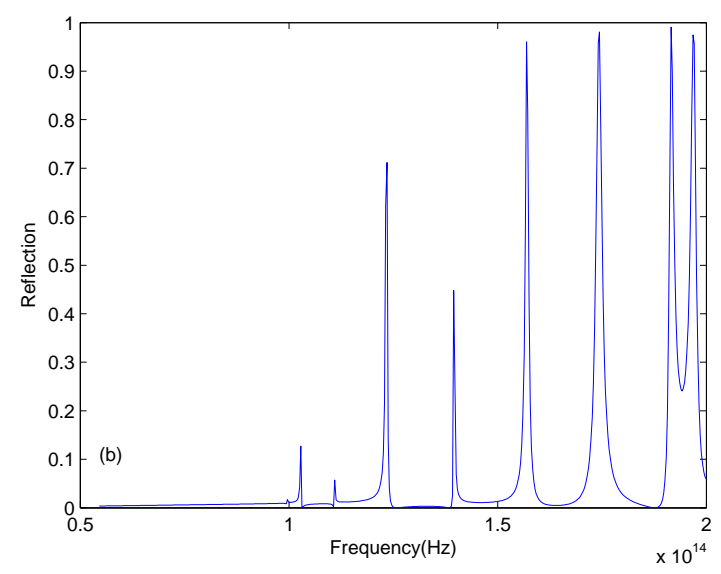

(b)

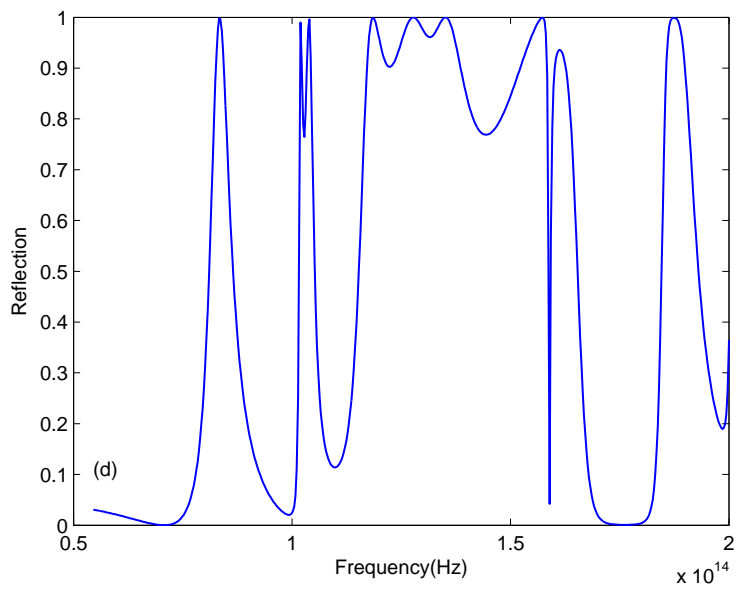

(d)

Fig. (2). Reflection spectra at normal incidence of the periodic dielectric periodic annular nanorod grating with the inner radius $R_{2}=0$ and the outer radius (a) $R_{l}=200 \mathrm{~nm}$, (b) $R_{l}=300 \mathrm{~nm}$, (c) $R_{l}=500 \mathrm{~nm}$, (d) $R_{l}=700 \mathrm{~nm}$, respectively.

\section{RESULTS AND DISCUSSION}

Parameter and angle resolved absolute reflection $(\mathrm{R})$ simulations are performed using the FDTD method. At normal incidence sharp reflection peaks are visible in the investigated frequency range when the inner radius of the nanorod is $R_{2}=0$, and the outer radius is small (a solid dielectric cylinder) (as shown in Fig. 1). For example, in Fig. (2a), the reflection characteristics indicate that in a broad frequency range, light is transmitted absolutely, however, at a very sharp frequency range, light is reflected nearly completely. For example, the value of the center peak the reflection spectrum is about 0.9 at the frequency $f=1.584 \times 10^{14} \mathrm{~Hz}$ with a very narrow width $\left(\Delta f=1.5869 \times 10^{14}-1.5811 \times 10^{14}=0.0058\right.$ $\times 10^{14} \mathrm{~Hz}$, where $\Delta f$ is the pass band width) (as shown in Fig. 2a)), which means that light is mostly transmitted in a wide range, but it is reflected absolutely in several narrow frequency ranges. In addition, it is shown that the resonance mode with a narrow line width, a high quality factor (High$\mathrm{Q})$, where $Q=\lambda_{0} / \Delta \lambda, \lambda_{0}$ is the wavelength of transmission peak and $\Delta \lambda$ is the full width at half maximum, for example, $Q=273.10$ for the structure with nanorod $R_{2}=200 \mathrm{~nm}$ for the reflection peak with center frequency $f=1.584 \times 10^{14} \mathrm{~Hz}$.
It indicates that the dielectric nanorod has a better frequency selecting characteristic and less absorbance than that of subwavelength metal gratings, especially for ones with small sizes. According to the formulated above, the reflection resonance modes sensitively depend on the radius of the nanorods with a high $Q$ factor, and the radius of the nanorod can be used to control the number and resonant frequency of the reflection resonance modes.

The reflection of the system can be considered as two components: Fano line shape induced by constructive and destructive interferences of direct transmission and light scattering by the nanorods [12]. The physical mechanism of this geometric resonance is closely related to a multiple scattering mechanism. Each subwavelength rod behaves like an individual scatter with a dipolar response. It can be modeled as an infinite circular cylinder with a polarizability, $\alpha \approx \alpha_{0}\left(1-i_{4}^{k^{2}} \alpha_{0}\right)^{-1}[13,15,18]$, where $\alpha_{0}=\pi R^{2}(\varepsilon-1)$ is the static polarizability, $R$ is the cylinder radius, and $\varepsilon$ is the dielectric permittivity of the rods. When illuminated by a plane wave, each rod of the array re-radiates a field 


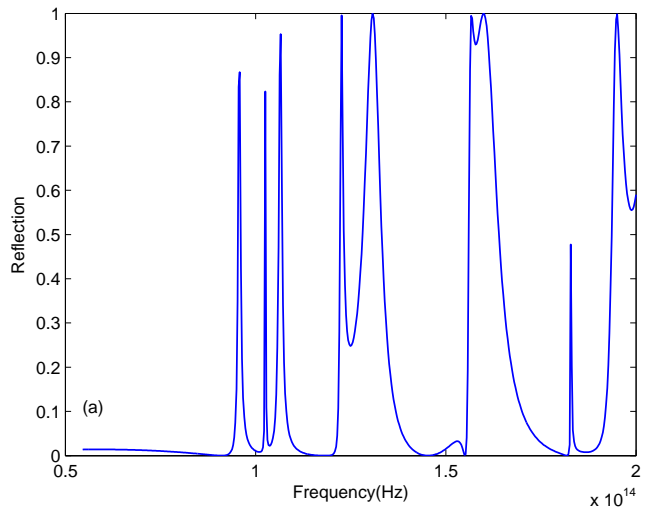

(a)

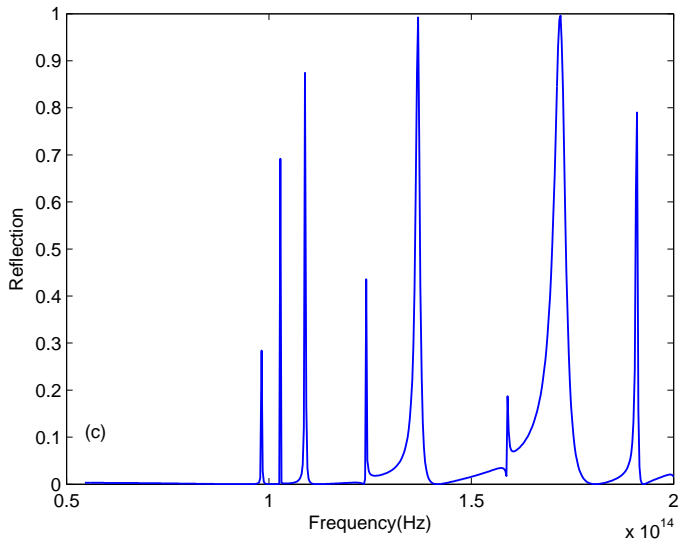

(c)

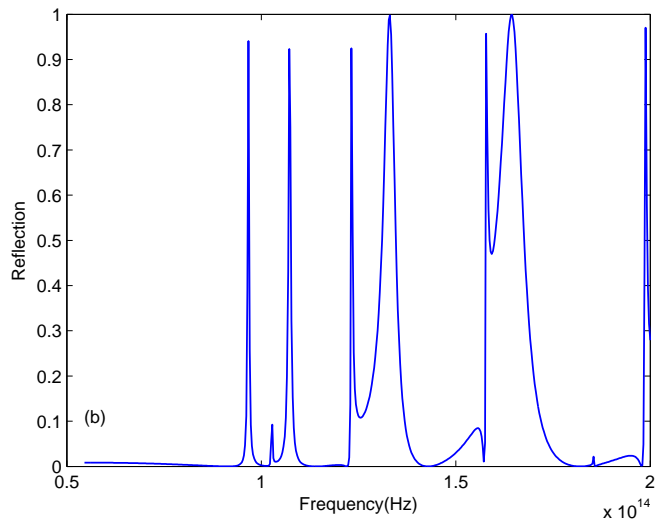

(b)

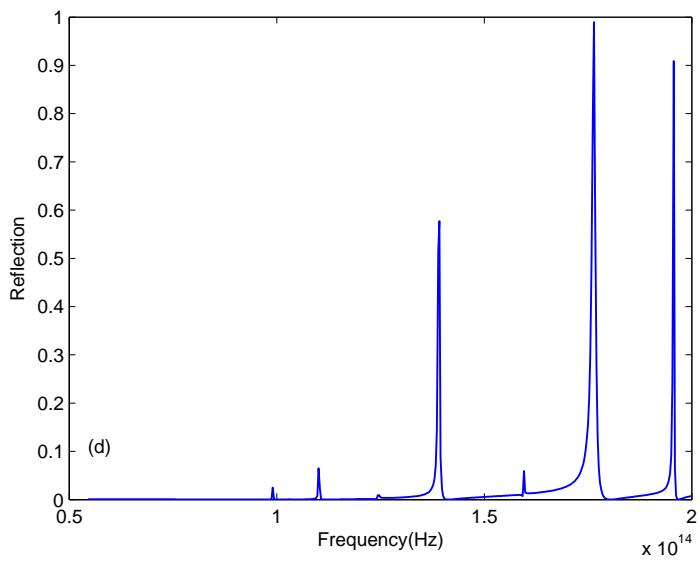

(d)

Fig. (3). Reflection spectra at normal incidence of the periodic dielectric periodic annular nanorod grating with the outer radius $R_{l}=500 \mathrm{~nm}$ and the inner radius (a) $R_{2}=200 \mathrm{~nm}$, (b) $R_{2}=300 \mathrm{~nm}$, (c) $R_{2}=400 \mathrm{~nm}$, and (d) $R_{2}=450 \mathrm{~nm}$, respectively.

proportional to its dipole moment $P_{y}$. Conversely, the incident field on each rod is the sum of the incident plane wave $\left(E_{i}\right)$ and the scattered fields $\left(E_{s}\right)$ from the other rods. The dipolar moment can be written as $p_{y}=\hat{\alpha} E_{i}, \hat{\alpha}=\left(\frac{1}{\alpha}-G_{b} k^{2}\right)^{-1}$, where the effective polarizability $\hat{\alpha}$ accounts for the multiple scattering mechanism. $G_{b}$ is the dynamic depolarization term which only depends on the geometrical parameters, i.e., the period $p$, the radius $R$, as well as the wavelength and the angle of incidence $\theta$. The geometric resonance is defined by the pole of the effective polarizability. This description leads to an analytical model for the far-field response of the membrane $[12,13,24]$. For the cylinder array, for p-polarized waves, the reflection resonances have a geometrical origin. The existence of particle surface modes or plasmons would reflect itself in a resonant behavior of the bare polarizabilities would present sharp maxima and minima around each internal resonant frequency surface modes would then induce new peaks in the reflection, when the surface resonance frequency is close to a Rayleigh frequency, they would mix with geometrical resonances leading to more complex reflection patterns. Results shown in Fig. (2) are according to that presented in $[12,13,24]$. With the cylinder radius $R_{l}$ increasing, light transmitted is increasing, and in the reflection spectra there appear more reflection peaks. Additionally, it is found that with new reflection resonance modes appearing at both the lower and the higher frequency region, the resonance mode at about $f=1.5 \times 10^{14} \mathrm{~Hz}$ is always present accompanied by the widening of the peak widths (as shown in Figs. (2b-d). Behaviors here indicate that the localization and magnitude of the sharp resonance mode are dependent on the dielectric nanorod radius sensitively, which can provide a regulation factor of the transmission property of this dielectric structure.

In Fig. (2), we calculate the reflection behaviors with the outer radius $R_{l}$ of the annular dielectric nanorod changing when the inner radius $R_{2}$ is set as 0 , here the structure is a solid cylinder similar as the one in Ref. [12]. Ref. [12] presents a multiple scattering study of the periodic nanoarray's reflectance. The characteristic of the dependence of optical properties on wavelengths, geometrical parameters, and cylinder dielectric constant are analytically investigated for s- and p-polarized waves, and the reflectance sharp peaks are attributed to the Rayleigh frequencies. At the lowest resonance frequency, and in the absence of absorption, the wave is perfectly reflected even for vanishingly small cylinder radii. And the results in Fig. (2) are according to that in Ref. [12].

Meanwhile, we simulated the reflection characteristics of the annular dielectric nanorod along the inner radius $R_{2}$ altering when the outer radius $R_{l}$ is set as $500 \mathrm{~nm}$, as shown in Fig. (3). According to the pictures in Fig. (3), the number of sharp peaks in the reflection spectra decreases along with $R_{2}$ 




(a)

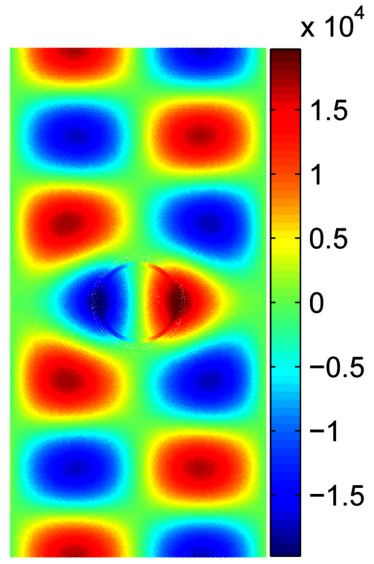

(b)

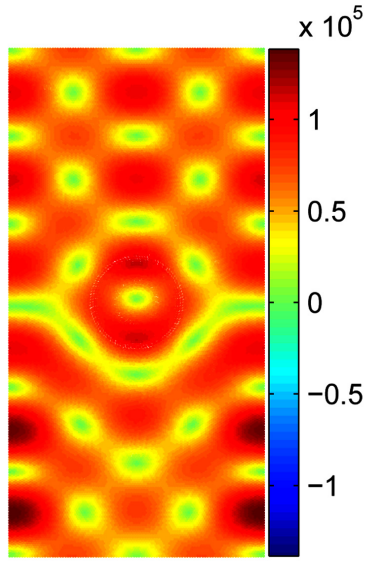

(c)

Fig. (4). Modulus distributions of the complex electric field (a) $E_{x}$, (b) $E_{y}$ and (c) normalized $E$, respectively, of the dielectric annular nanorod array with outer radius $R_{l}=500 \mathrm{~nm}$, inner radius $R_{2}=450 \mathrm{~nm}$, with a normal incidence at the resonance frequency $f=1.7643 \times 10^{14} \mathrm{~Hz}$.

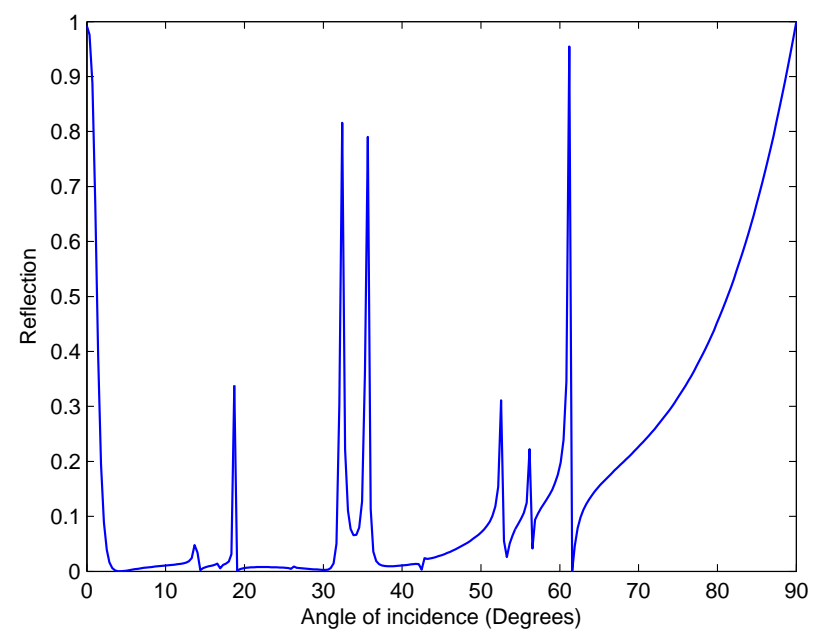

Fig. (5). Reflection spectra as a function of the incidence angle of the periodic dielectric periodic annular nanorod grating with outer radius $R_{1}=500 \mathrm{~nm}$, inner radius $R_{2}=450 \mathrm{~nm}$, at the center frequency of the reflection peak $f=1.7643 \times 10^{14} \mathrm{~Hz}$.

increasing; meanwhile, peak width is narrowed. For example, there are a series of reflection peaks in the spectrum with the inner radius $R_{2}=200 \mathrm{~nm}$, and three modes are left when $R_{2}$ is increased to $450 \mathrm{~nm}$. The result here means that the magnitude of geometric parameter inner radius of the annular dielectric nanorod can also modulate the resonance mode obviously. It is shown that nearly all light over a precise set of wavelength can be reflected. With increasing of the solid cylinder radius, more reflection peaks appear accompanied by the widening of the peak width, with increasing of the inner radius of the annular cylinder, the reflection peaks lessens gradually, meanwhile, the peak width are decreased distinctly, which means that identical properties can be obtained by increasing the inner radius of the annular one when the outer radius is fixed and by decreasing the radius of the solid cylinder. With inner radius is increasing, the annular thickness becomes thinner, more light can be transmitted and the reflection is weakened, which may be associated with the reflection peak lessening and the peak width narrowing. There would be more geometrical factors of the annular nanorods than that of the uniform homogeneous cylinders.
We can modulate the line shape not only by the size of the cylinder, the gap of the cylinder, the incidence angle, but also by the inner size of the annular cylinder.

In relation with the sharp reflection resonance, the nanorod array exhibits very high field enhancements. The maps of the electric field intensity $\mathrm{E}_{\mathrm{x}}, \mathrm{E}_{\mathrm{y}}$, and the normalized $\mathrm{E}$ have been calculated as shown in Fig. (4) for the 1 dimension array. It is found that the maximum and minimum of electric field distributes alternately as the existence of the nanorod. It is shown that there is no resonant coupling among the dipoles pointing along $\mathrm{X}$-axis. It can be associated with the reflection geometrical resonance $[12,15]$. Additionally, the reflection resonances manifest themselves in a resonant fluorescence enhancement near the nanostructures as discussed in Ref. [15].

Spectra were also recorded under an incidence angle $\theta$ ranging from $0^{\circ}$ to $90^{\circ}$ by $0.36^{\circ}$ increments, as shown in Fig. (5), for the annular dielectric nanorod array with the outer radius $R_{l}=500 \mathrm{~nm}$, inner radius $R_{2}=450 \mathrm{~nm}$, at the resonance frequency $f=1.7643 \times 10^{14} \mathrm{~Hz}$. Reflection is about 0.9 when 


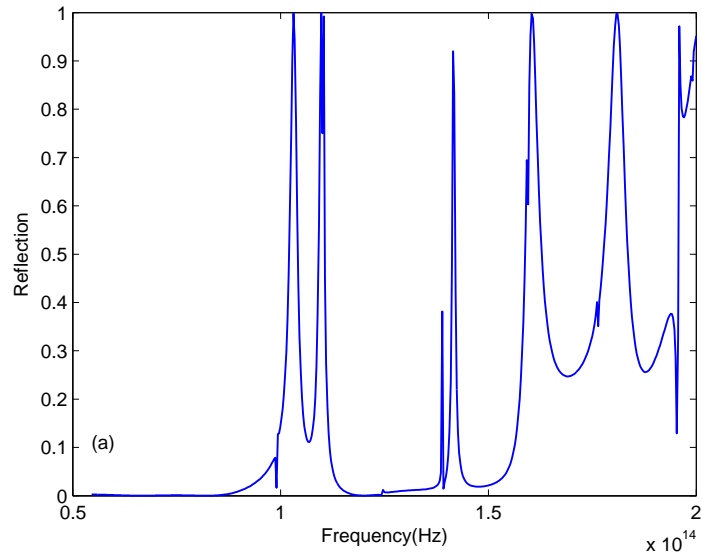

(a)

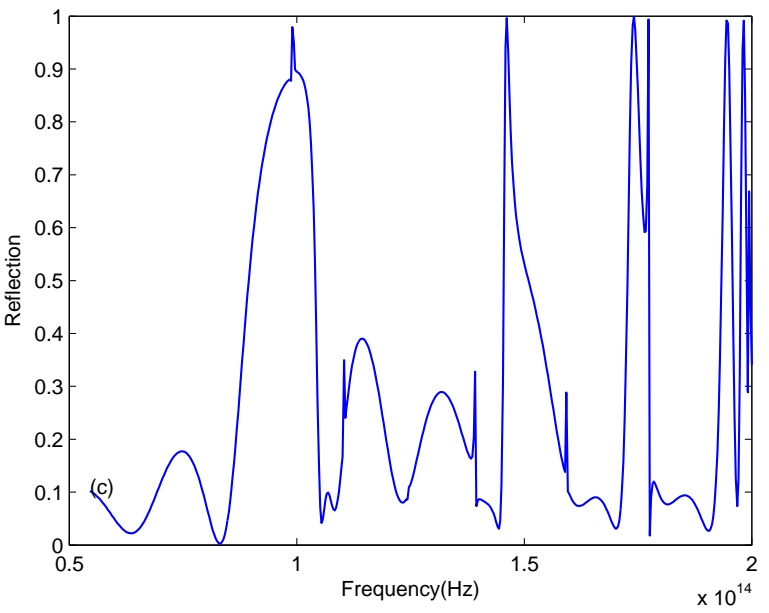

(c)

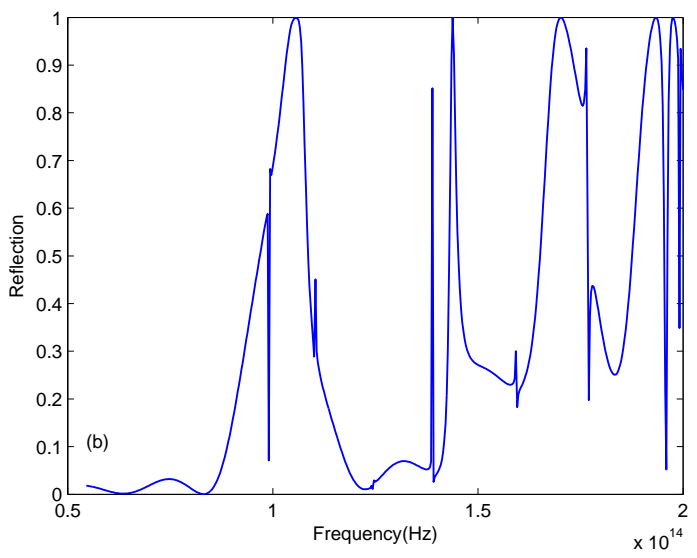

(b)



(d)

Fig. (6). Reflection spectra of the periodic dielectric periodic annular nanorod grating with outer radius $R_{l}=500 \mathrm{~nm}$, inner radius $R_{2}=450 \mathrm{~nm}$ for incidence angle (a) $\theta=20^{\circ}$, (b) $\theta=40^{\circ}$, (c) $\theta=60^{\circ}$, and (d) $\theta=80^{\circ}$, respectively.

the incidence angel $\theta=0^{\circ}$, the reflection decreases firstly as a function of the angle of incidence at which it is less than $10^{\circ}$. For example, when the incidence angle reaches about $5^{\circ}$, nearly all the light passes through, with a reflection of only 0.001 . In the angle region between $20^{\circ}$ and $60^{\circ}$, most light is transmitted with only several narrow bands reflected. Reflection increases gradually to 1 as the incidence angle rises further to $90^{\circ}$.

Fig. (6) shows the results of the structure with four different angles of incident $20^{\circ}, 40^{\circ}, 60^{\circ}, 80^{\circ}$, respectively, with outer radius $R_{I}=500 \mathrm{~nm}$, inner radius $R_{2}=450 \mathrm{~nm}$ of the annular dielectric nanorod array. The modulations of the maximum of the reflection correspond to the transparency and absorption bands of the material. Line widths and shapes of the reflection resonance peaks are alternated dramatically with the change of the incidence angle. For example, with the incidence angle increasing, more reflection resonance modes appear, as shown in Figs. (6a-d). Additionally, as the degree increases to $90^{\circ}$, nearly all the light is reflected, and almost no light is transmitted. It originates from multiple scattering involving the excitation of the dipole moment.

\section{CONCLUSION}

In conclusion, we simulate the optical property of an array of annular dielectric nanorods. In absence of Mie resonances and surface (plasmon) modes, the reflectance presents sharp peaks close to the onset of new diffraction modes. At the lowest resonance frequency, and in the absence of absorption, the wave is perfectly reflected even for vanishingly small cylinder radii. It is shown that nearly all light over a precise set of wavelength can be reflected, With increasing of the solid cylinder radius, more reflection peaks appear accompanied by the widening of the peak width, with increasing of the inner radius of the annular cylinder, the reflection peaks lessen gradually, meanwhile, the peak width are decreased distinctly. With outer or inner radius changing, the reflection peak at around $f=1.5 \times 10^{14} \mathrm{~Hz}$ is always present. The optical properties dependences on incidence angle are analytically derived. The annular dielectric cylinder array can provide more modulating factors of the reflection spectra such as the inner radius than that with solid cylinders. This phenomenon is associated with that the scatters are nonresonant and much smaller than the wavelength and free-space interactions between the scatters. These 
results are helpful for the design of novel optical device by using such dielectric material.

\section{CONFLICT OF INTEREST}

The authors confirm that this article content has no conflict of interest.

\section{ACKNOWLEDGMENTS}

Supported by National Natural Science Foundation of China (under grant: 11304094), the Hunan Provincial Natural Science Foundation of China (under grant: 12JJB001), and the Scientific Research Fund of Hunan Provincial Education Department (under grant: 13C322).

\section{REFERENCES}

[1] Ebbesen TW, Lezec HJ, Ghaemi HF, Thio T, Wolff PA. Extraordinary optical transmission through sub-wavelength hole arrays. Nature (London) 1998; 391: 667-9.

[2] Liu H, Lalanne P. Microscopic theory of the extraordinary optical transmission. Nature (London) 2008; 452: 728-31.

[3] Kravets VG, Schedin F, Grigorenko AN. Extremely narrow plasmon resonances based on diffraction coupling of localized plasmons in arrays of metallic nanoparticles. Phys Rev Lett 2008; 101: 087403.

[4] Kulkarni GU, Radha B. Metal nanowire grating patterns. Nanoscale 2010; 2: 2035-44.

[5] Zhu YJ, Huang XG, Mei X. A surface plasmon polariton electrooptic switch based on a metal-insulator-metal structure with a strip waveguide and two side-coupled cavities. Chin Phys Lett 2012; 29: 064214.

[6] Zhong YT, Cheng ZQ, Ma L, Wang JH, Hao ZH, Wang QQ. Surface plasmon resonance and Raman scattering activity of the $\mathrm{Au} / \mathrm{Ag}_{\mathrm{x}} \mathrm{O} / \mathrm{Ag}$ multilayer film. Chin Phys Lett 2014; 31: 047302.

[7] Zolanvari A, Sadeghi H, Norouzi R, Ranjgar A. Surface plasmons and optical properties of $\mathrm{TiO}_{2} / \mathrm{X}(\mathrm{X}=\mathrm{Au}$ and $\mathrm{Ag})$. Chin Phys Lett 2013; 30: 096201

[8] Kitazawa T, Miyanishi S, Murakami Y, Kojima K, Takahashi A. Snom observations of surface plasmon polaritons on metal heterostructures. Chin Phys Lett 2007; 24: 2827.
Zou S, Janel N, Schatz GC. Silver nanoparicle array structures that produce remarkably narrow plasmon lineshapes. J Chem Phys 2004; 120: 10871.

[10] Markel VA. Divergence of dipole sums and the nature of nonlorentizian exponentially narrow resonances in one dimensional periodic arrays of nanospheres. J Phys B 2005; 38: L115-21.

[11] de Abajo FJG. Colloquium: Light scattering by particle and hole arrays. Rev Mod Phys 2007; 79: 1267.

[12] Gomez-Medina R, Laroche M, Saenz JJ. Extraordinary optical reflection from sub-wavelength cylinder arrays. Opt Express 2006; 14: 3730.

[13] Laroche M, Albaladejo S, Gomez-Medina R, Saenz JJ. Tuning the optical response of nanocylinder arrays: an analytical study. Phys Rev B 2006; 74: 245422.

[14] Kottmann JP, Martin OJF, Smith DR, Schultz S. Quantum and transport conductivities in monolayer grapheme. Phys Rev B 2001; 64: 235402 .

[15] Kuhn S, Hakanson U, Rogobete L, Sandoghdar V. Enhancement of single-molecule fluorescence using a gold nanoparticle as an optical nanoantenna. Phys Rev Lett 2006; 97: 017402.

[16] Danckwerts M, Novotny L. Optical frequency mixing at coupled gold nanoparticles. Phys Rev Lett 2007; 98: 026104.

[17] Greffet JJ, Carminati R, Joulain K, Mulet JP, Mainguy S, Chen Y. Coherent emission of light by thermal sources. Nature (London) 2002; 416: 61-4

[18] Collin S, Vincent G, Haidar R, Bardou N, Rommeluere S, Pelouard JL. Nearly perfect Fano transmission resonances through nanoslits drilled in a metallic membrane. Phys Rev Lett 2010; 104: 027401.

[19] Haidar R, Vincent G, Collin S, et al. Achievement of ultrahigh quality factors in GaAs photonic crystal membrane nanocavity. Appl Phys Lett 2010; 96: 221104.

[20] Kippenberg TJ, Vahala KJC. Cavity optomechanics: Back-action at the mesoscale. Science 2008; 321: 1172-6.

[21] Thompson JD, Zwickl BM, Jayich AM, Marquardt F, Girvin SM, Harris JGE. Strong dispersive coupling of a high-finesse cavity to a micromechanical membrane. Nature (London) 2008; 452: 72-5.

[22] Yee KS. Numerical solution of initial boundary value problems involving Maxwell's equations in isotropic media. IEEE Trans Antennas Propag 1966; 14: 302-7.

[23] Berenger JP. A perfectly matched layer for the absorption of electromagnetic waves. J Comput Phys 1994; 114: 185-200.

[24] Ghenuche P, Vincent G, Laroche M, Bardou N, Haidar R. Optical extinction in a single layer of nanorods. Phys Rev lett 2012; 109: 143903.

(C) Xie et al.; Licensee Bentham Open.

This is an open access article licensed under the terms of the Creative Commons Attribution Non-Commercial License (http://creativecommons.org/licenses/by-nc/3.0/) which permits unrestricted, non-commercial use, distribution and reproduction in any medium, provided the work is properly cited. 